\section{The NOvA Detectors}

NOvA is a long-baseline neutrino oscillation experiment based at Fermilab that uses the NuMI beam line and two detectors to measure neutrino rates at a near location, and 810 $\mathrm{km}$ away at a far location. made from PVC extrusions which segment the

volume in to $4 \times 6 \mathrm{~cm}$ cells which alternate orientations to provide $3 \mathrm{D}$ tracking of particles in the detector.

\section{Neutrino Tridents}

Neutrino tridents are a rare Standard Model process in which a neutrino interacts with a nucleus and produces two charged leptons. Dimuon tridents have been observed by multiple collaborations including CHARM-II [1], CCFR [2], and NuTeV [3]. The high Near Detector makes a search viable. The interaction may be mediated by a Z' boson

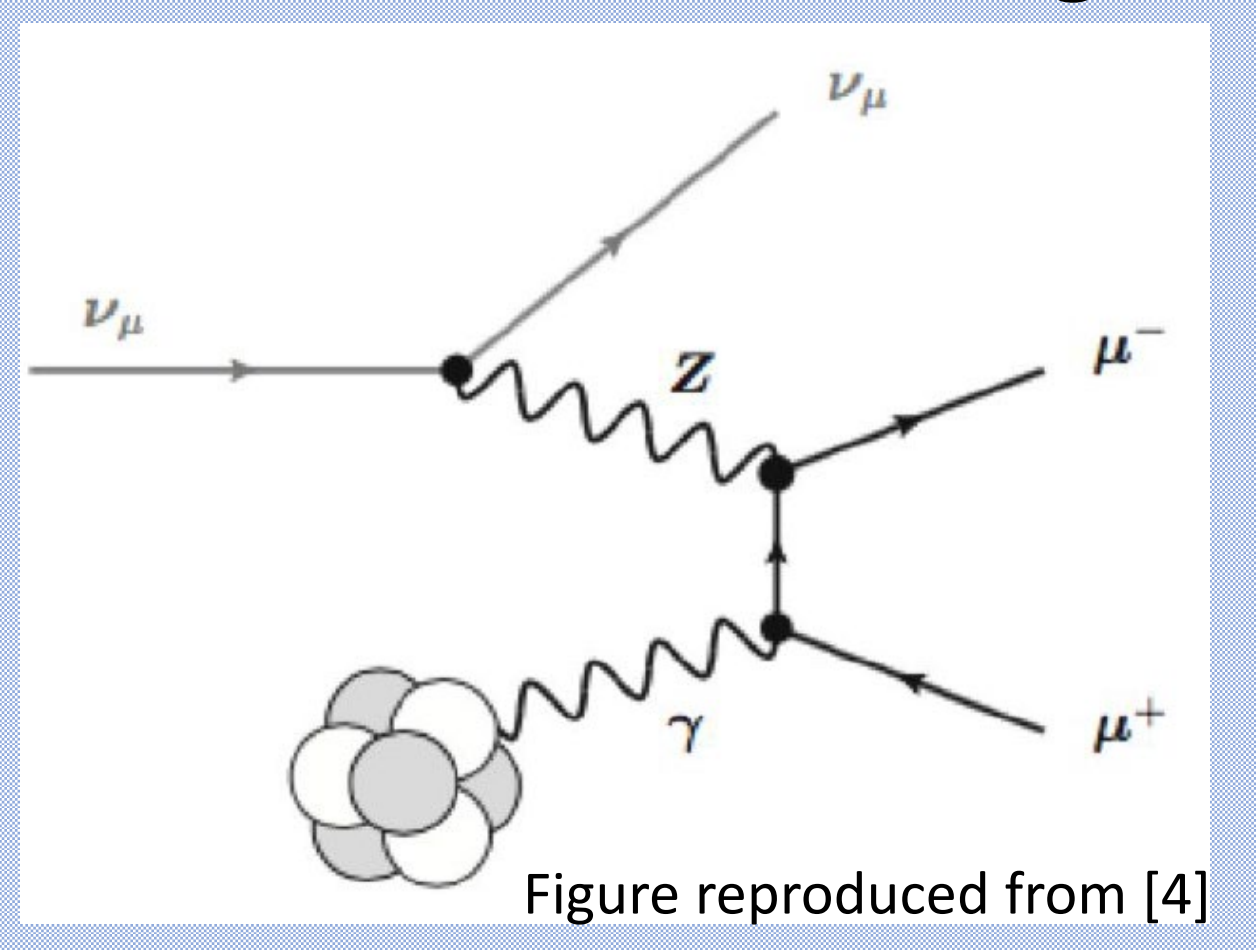
whose addition to the Standard Model could aid the understanding of the anomalous magnetic moment of the muon. The detectors are neutrino flux at the NOvA

\section{Simulation}

To simulate neutrino trident events in the NOvA Near Detector, we adapted the simulation provided by Altmannshofer et al. [4] by importing the NOvA beam flux, assigning events a random position, and importing the structure functions for carbon. We estimate that NOvA should see 10-100 trident events per year, somewhat higher than other estimates [5].

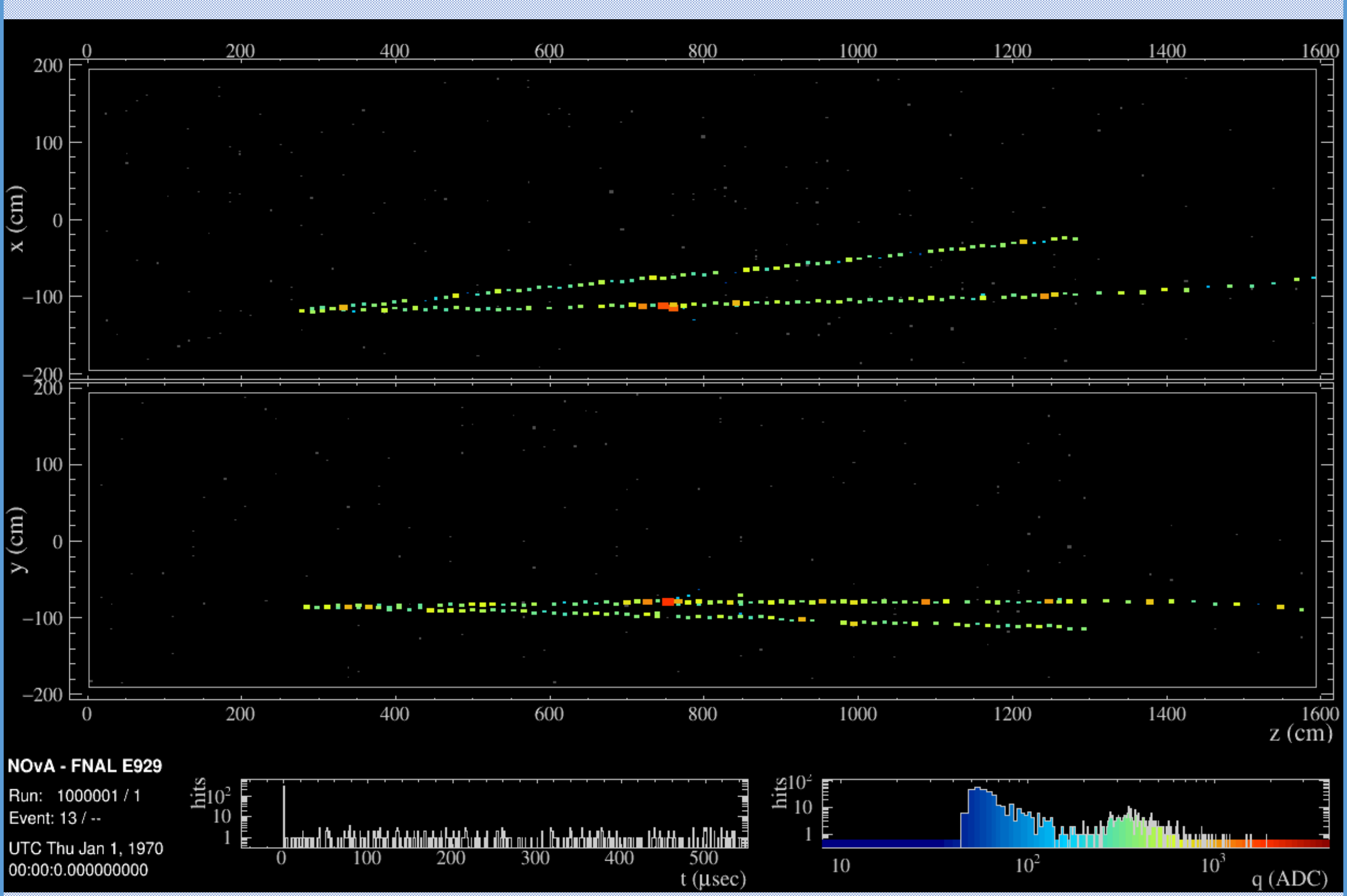

An example trident in the NOvA Near Detector is shown above. Dimuon trident events are expected to have two energetic muons and low hadronic energy at the vertex.

If you're interested in tridents, you may also like: $M$. Judah, The measurement of the inclusive electron-neutrino charged-current double-differential cross-section using the NOvA near detector

C. Sweeney, Measuring cross sections of neutrino-nucleus interactions with associated charged pions in the NOvA near detector

C. Johnson, Muon-neutrino charged-current inclusive cross-sections using the NOvA near detector

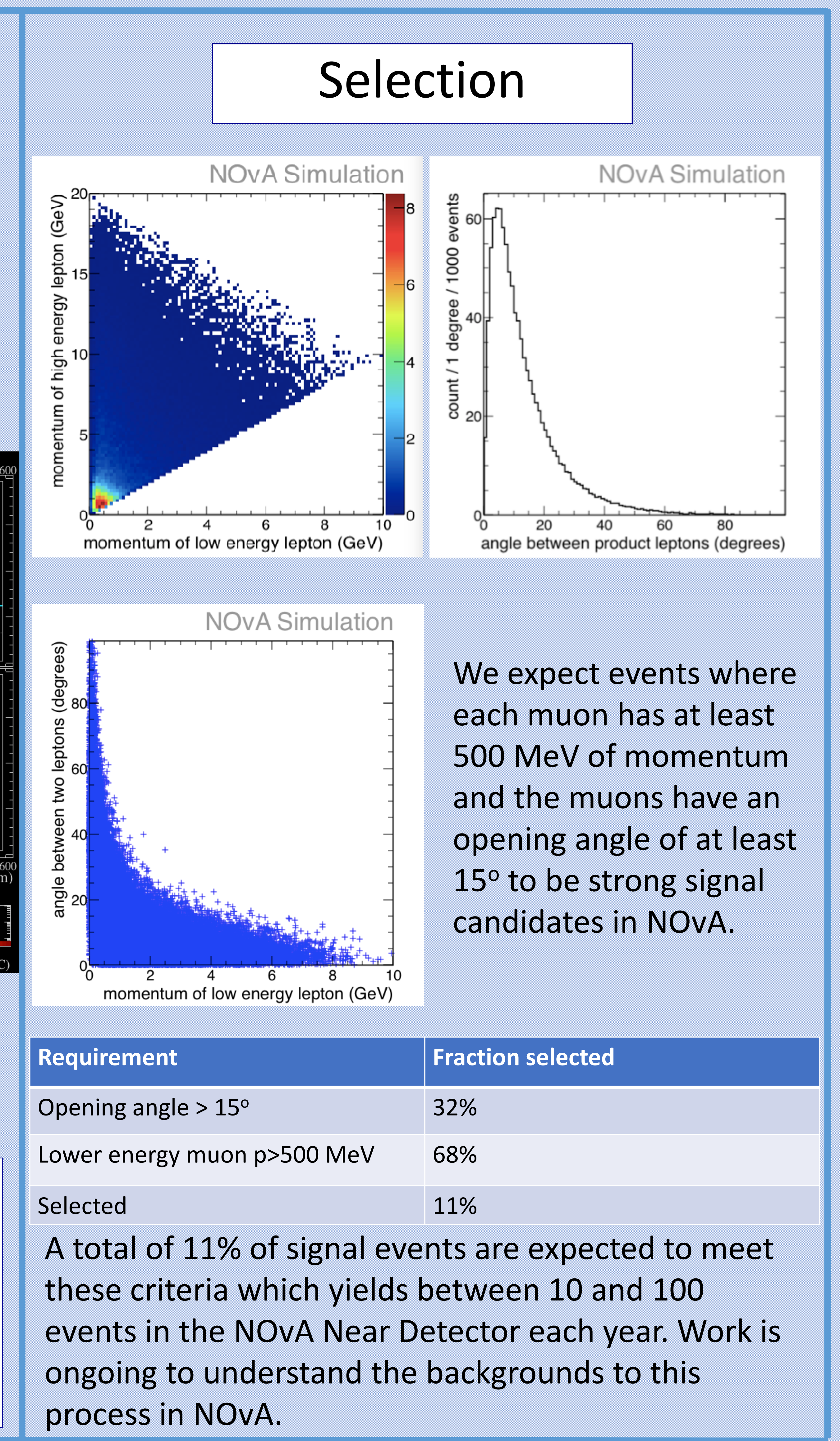

[1] D. Geiregat et al. (CHARM-II Collaboration), Phys. Lett. B 245 271, (1990). [2] S. R. Mishra et al. (CCFR Collaboration), Phys. Rev. Lett. 66, 3117 (1991). [3] T. Adams et al. (NuTeV Collaboration), Phys. Rev. D 61, 092001 (2000). [4] W. Altmannshofer et al., Phys. Rev. D 100, 115029 (2019). [5] P. Ballett et al., JHEP 01119 (2019).
This work is supported by:

- Fermi National Accelerator Laboratory

- US Department of Energy
This document was prepared by NOvA collaboration] using the resources of the Office of Science, HEP User Facility. Fermilab is managed by Fermi Research Alliance, LLC (FRA), acting under Contract No. DE-AC02-07CH11359.

http://novaexperiment.fnal.gov Fermi National Accelerator Laboratory (Fermilab), a U.S. Department of Energy, 